Research Article

\title{
Modelling and Analysis of Propagation Behavior of Computer Viruses with Nonlinear Countermeasure Probability and Infected Removable Storage Media
}

\author{
Xulong Zhang $(\mathbb{D}$ and Yong Li (iD \\ School of Computer and Network Engineering, Shanxi Datong University, Datong 037009, China \\ Correspondence should be addressed to Xulong Zhang; zxl-095@163.com
}

Received 13 August 2020; Revised 22 September 2020; Accepted 30 September 2020; Published 26 October 2020

Academic Editor: Jianxin Li

Copyright ( 2020 Xulong Zhang and Yong Li. This is an open access article distributed under the Creative Commons Attribution License, which permits unrestricted use, distribution, and reproduction in any medium, provided the original work is properly cited.

\begin{abstract}
The dissemination of countermeasures is diffusely recognized as one of the most valid strategies of containing computer virus diffusion. In order to better understand the impacts of countermeasure and removable storage media on viral spread, this paper addresses a dynamical model, which incorporates nonlinear countermeasure probability and infected removable storage media. Theoretical analysis reveals that the unique (viral) equilibrium of the model is globally asymptotically stable. This main result is also illustrated by some numerical experiments. Additionally, the numerical experiments of different countermeasure probabilities are conducted.
\end{abstract}

\section{Introduction}

The continual emergence of computer viruses, especially with the growing popularity of the Internet, has brought great troubles and threats to our daily work and life (e.g., [1]). Besides, removable storage media, such as compact disk, removable hard disk, USB flash disk, flash memory card, and so on, which are often used in our daily work provide another spreading route for computer viruses except the Internet. Antivirus software, patches, and firewall are the main technical means of defending against computer viruses, which can weed out all viruses they can recognize that stay in individual electronic devices such as personal computer (PC) and removable storage media. Unfortunately, these techniques seem powerless to the outbreak of a new virus. In order to effectively contain virus spread, one needs to understand the propagation laws of computer viruses, which may provide a theoretical basis for decision making, as well as to use technical measures.

A multitude of propagation models of computer viruses have been presented since 1991, specifically, SIS (susceptibleinfected-susceptible) models (e.g., [2,3]), SIRS (susceptible- infected-recovered-susceptible) models (e.g., $[4,5])$, SLBS (susceptible-latent-breaking-susceptible) model (e.g., [6]), SICS (susceptible-infected-countermeasured-susceptible) models (e.g., [7-9]), and SDIRS (susceptible-delitescentinfected-recovered- susceptible) model (e.g., [10]).

In the field of computer viruses, countermeasures such as warnings, firewall, and software patches can provide a practical approach to avoid virus infection problems. In 2004, Chen and Carley [11] addressed the countermeasure competing (CMC) strategy, which shows that the CMC strategy is more available compared to previous strategies.

Inspired by this work and in order to macroscopically describe the mixed transmission of computer viruses and countermeasures, Zhu et al. [7] presented the first compartment model in this aspect, named as the SICS model, and its global dynamics was fully examined. Afterwards, Yang and Yang [8] extended this model by incorporating the effect of infected external computers (i.e., computers outside the Internet) and removable storage media. However, these two models ignore two important facts. On the one hand, they overlook the fact that the linear infection probability is fit well for the real-world situations only when the 
countermeasured (or immune) nodes are few. On the other hand, they neglect the fact that countermeasures may be disseminated through networks at different rates, which has been mentioned in Reference [11]. Thus, the assumption of linear countermeasure probability is unreasonable.

To remedy these defects and considering the influences of general countermeasure and infected removable storage media on viral diffusion, this paper studies a new propagation model incorporating generic countermeasure probability and infected removable storage media. The main result, the global stability of the unique (viral) equilibrium, is proved, which is also examined by some numerical experiments. Furthermore, the numerical experiments of different countermeasure probabilities are conducted.

The paper is organized as follows. The model formulation is made in Section 2. Section 3 determines the (viral) equilibrium and investigates its global stability. Numerical experiments are presented in Section 4. This work is summarized in Section 5.

\section{Model Description}

In this paper, a computer is called external or internal computer determined by whether it is disconnected from or connected to the Internet. All internal computers may have three states: susceptible, infected, and immune (with countermeasures). For brevity, let $S(t), I(t)$, and $C(t)(S, I$, and $C$, for short) denote the average numbers of susceptible, infected, and countermeasured computers at time $t$, respectively. Their entering rates are $\mu_{1}>0, \mu_{2}>0$, and $\mu_{3}>0$, respectively. Besides, the following basic assumptions of the model are made.

(1) Each internal computer leaves the Internet with probability $\delta>0$.
(2) Each susceptible internal computer becomes infected by connecting with infected internal computer (or infected removable storage media) with probability $\beta_{1}>0$ (or $\beta_{2}>0$ ).

(3) Each infected or susceptible internal computer gains the latest countermeasure with probability $\gamma_{1}(C(t))$ at time $t$, where $\gamma_{1}$ is twice continuously differentiable, $\gamma_{1}^{\prime}>0, \gamma_{1}^{\prime \prime}<0$, and $\gamma_{1}(0)=0$. The concavity assumption seizes well the saturability of the countermeasure probability.

(4) By reinstalling the operating system, each countermeasured (or infected) internal computer becomes susceptible with probability $\alpha>0$ (or $\gamma_{2}>0$ ).

Now, one can derive the mathematical representation of the model as follows (also see Figure 1):

$$
\left\{\begin{array}{l}
\frac{\mathrm{d} S}{\mathrm{~d} t}=\mu_{1}-\beta_{1} S I-\beta_{2} S-\gamma_{1}(C) S+\gamma_{2} I+\alpha C-\delta S, \\
\frac{\mathrm{d} I}{\mathrm{~d} t}=\mu_{2}+\beta_{1} S I+\beta_{2} S-\gamma_{1}(C) I-\gamma_{2} I-\delta I, \\
\frac{\mathrm{d} C}{\mathrm{~d} t}=\mu_{3}+\gamma_{1}(C) S+\gamma_{1}(C) I-\alpha C-\delta C,
\end{array}\right.
$$

with initial condition $(S(0), I(0), C(0)) \in \mathbb{R}_{+}^{3}$.

\section{Theoretical Analysis}

Let $N=S+I+C$, and $\mu=\mu_{1}+\mu_{2}+\mu_{3}$. Adding up the three equations of system (1), it is easy to get that $\lim _{t \rightarrow \infty} N=(\mu / \delta)$. It follows by the asymptotically autonomous system theory [12] that system (1) is equivalent to the following reduced limiting system:

$$
\left\{\begin{array}{l}
\frac{\mathrm{d} I}{\mathrm{~d} t}=\mu_{2}+\frac{\beta_{2} \mu}{\delta}+\left(\frac{\beta_{1} \mu}{\delta}-\beta_{2}-\gamma_{2}-\delta\right) I-\beta_{2} C-\beta_{1} I^{2}-\beta_{1} I C-\gamma_{1}(C) I \\
\frac{\mathrm{d} C}{\mathrm{~d} t}=\mu_{3}+\gamma_{1}(C)\left(\frac{\mu}{\delta}-C\right)-(\alpha+\delta) C
\end{array}\right.
$$

with initial condition $(I(0), C(0)) \in \Omega$, where

$$
\Omega=\left\{(I, C) \in \mathbb{R}_{+}^{2}: I+C \leq \frac{\mu}{\delta}\right\},
$$

and $\Omega$ is positively invariant for system (2).

In the following sections, we just need to investigate the dynamical behavior of system (2).

\subsection{Equilibrium}

Theorem 1. There exists a unique (viral) equilibrium $E^{*}=$ $\left(I^{*}, C^{*}\right)$ for system (2), where $E^{*}$ is the single positive solution to the following system:

$$
\left\{\begin{array}{l}
\mu_{2}+\frac{\beta_{2} \mu}{\delta}+\left(\frac{\beta_{1} \mu}{\delta}-\beta_{2}-\gamma_{2}-\delta\right) x-\beta_{2} y-\beta_{1} x^{2}-\beta_{1} x y-\gamma_{1}(y) x=0, \\
\mu_{3}+\gamma_{1}(y)\left(\frac{\mu}{\delta}-y\right)-(\alpha+\delta) y=0,
\end{array}\right.
$$




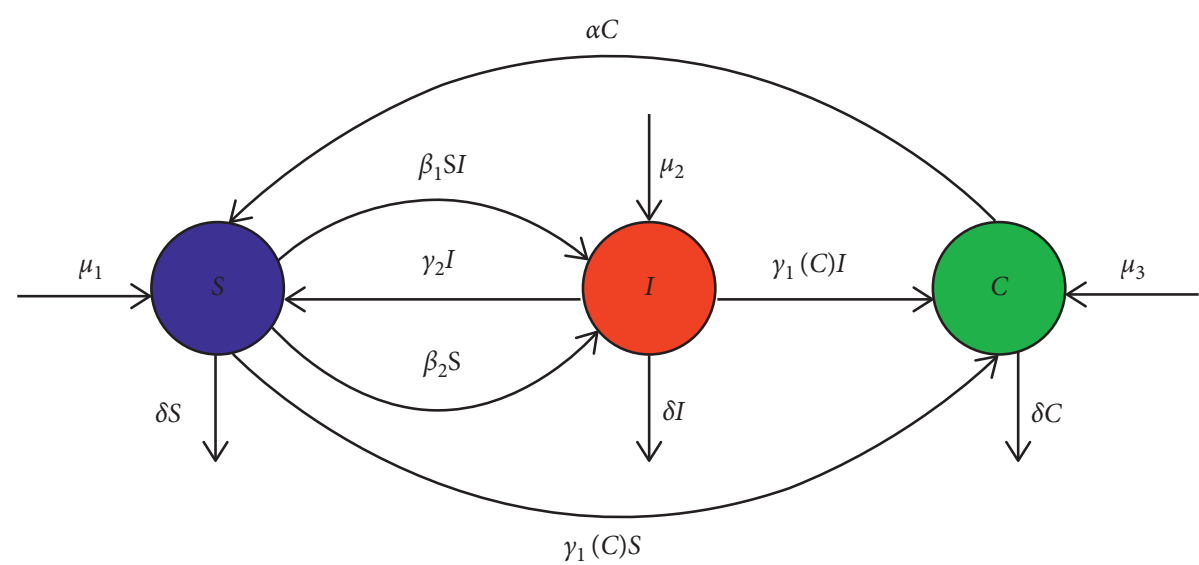

Figure 1: Transfer diagram of the new proposed model.

with the initial condition $(x(0), y(0)) \in \Omega$.

Proof. Let us suppose that $E^{*}=\left(I^{*}, C^{*}\right)$ is an equilibrium of system (2). Clearly, $E^{*}$ satisfies system (4). Thus, it suffices to prove that system (4) has a unique positive solution.

Firstly, let us prove that the second equation of system (4) has a unique positive root. Let

$$
f(y)=\mu_{3}+\gamma_{1}(y)\left(\frac{\mu}{\delta}-y\right)-(\alpha+\delta) y .
$$

As $f(0)=\mu_{3}>0$ and $f(\mu / \delta)=-(\alpha \mu / \delta)-\left(\mu_{1}+\mu_{2}\right)<0$, so $f$ does have a zero located in $(0,(\mu / \delta))$. Besides, notice that

$$
\begin{aligned}
& f^{\prime}\left(\frac{\mu}{\delta}\right)=-\gamma_{1}\left(\frac{\mu}{\delta}\right)-(\alpha+\delta)<0, \\
& f^{\prime \prime}(y)=\gamma_{1}^{\prime \prime}(y)\left(\frac{\mu}{\delta}-y\right)-2 \gamma_{1}^{\prime}(y)<0 .
\end{aligned}
$$

We shall consider two possibilities depending upon whether $f^{\prime}(0)$ is positive or negative.

Case 1: $f^{\prime}(0)>0$. Let

$$
\bar{y}=\max \left\{y \in\left[0, \frac{\mu}{\delta}\right]: f^{\prime}(y)>0\right\} .
$$

Thus, $f$ is strictly increasing in $[0, \bar{y}]$ and strictly decreasing in $[\bar{y},(\mu / \delta)]$, which implies that $f$ has a unique zero in $[\bar{y},(\mu / \delta)]$.

Case 2: $f^{\prime}(0) \leq 0$. So, $f$ is decreasing and has a unique zero.

It is easily obtained from the above discussions that $f$ does has a single zero. Then, $y=C^{*}$. Besides, $f^{\prime}\left(C^{*}\right)<0$.

Next, let us prove that the first equation of system (4) has a single positive root. Let

$$
\begin{aligned}
g(x)= & \mu_{2}+\beta_{2}\left(\frac{\mu}{\delta}-C^{*}\right)+\left(\frac{\beta_{1} \mu}{\delta}-\beta_{2}-\gamma_{2}-\delta-\beta_{1} C^{*}\right. \\
& \left.-\gamma_{1}\left(C^{*}\right)\right) x-\beta_{1} x^{2} .
\end{aligned}
$$

As $g(0)=\mu_{2}+\beta_{2}\left((\mu / \delta)-C^{*}\right)>0$ and $g\left((\mu / \delta)-C^{*}\right)=$ $-\mu_{1}-\alpha C^{*}-\gamma_{2}\left((\mu / \delta)-C^{*}\right)<0, g$ does have a (positive) zero located in $\left(0,(\mu / \delta)-C^{*}\right)$. Besides, note that

$$
\begin{aligned}
g^{\prime}\left(\frac{\mu}{\delta}-C^{*}\right) & =-\beta_{1}\left(\frac{\mu}{\delta}-C^{*}\right)-\left(\beta_{2}+\gamma_{2}+\delta+\gamma_{1}\left(C^{*}\right)\right)<0, \\
g^{\prime \prime}(x) & =-2 \beta_{1}<0 .
\end{aligned}
$$

We shall also proceed by distinguishing two possibilities depending upon whether $g^{\prime}(0)$ is positive or negative.

Case 1: $g^{\prime}(0)>0$. Let

$$
\bar{x}=\max \left\{x \in\left[0, \frac{\mu}{\delta}-C^{*}\right]: g^{\prime}(x)>0\right\} .
$$

Then, $g$ is strictly increasing in $[0, \bar{x}]$ and decreasing in $\left[\bar{x},(\mu / \delta)-C^{*}\right]$, meaning that $g$ has a single zero in $\left[\bar{x},(\mu / \delta)-C^{*}\right]$.

Case 2: $g^{\prime}(0) \leq 0$. Hence, $g$ is decreasing and has a unique zero. Then, $g$ always has a single zero $x=I^{*}$. Besides, $g^{\prime}\left(I^{*}\right)<0$.

Thus, the claimed result follows.

\subsection{Local Stability}

Theorem 2. $E^{*}$ is locally asymptotically stable. 
Proof. The corresponding Jacobian matrix of system (2) at $E^{*}$ is as follows:

$$
\left(\begin{array}{cc}
\frac{\beta_{1} \mu}{\delta}-\beta_{2}-\gamma_{2}-\delta-\beta_{1} C^{*}-2 \beta_{1} I^{*}-\gamma_{1}\left(C^{*}\right) & -\beta_{2}-\beta_{1} I^{*}-\gamma_{1}^{\prime}\left(C^{*}\right) I^{*} \\
0 & \gamma_{1}^{\prime}\left(C^{*}\right)\left(\frac{\mu}{\delta}-C^{*}\right)-\gamma_{1}\left(C^{*}\right)-(\alpha+\delta)
\end{array}\right)
$$

and its two eigenvalues are

$$
\begin{aligned}
& \lambda_{1}=\frac{\beta_{1} \mu}{\delta}-\beta_{2}-\gamma_{2}-\delta-\beta_{1} C^{*}-2 \beta_{1} I^{*}-\gamma_{1}\left(C^{*}\right)=g^{\prime}\left(I^{*}\right)<0, \\
& \lambda_{2}=\gamma_{1}^{\prime}\left(C^{*}\right)\left(\frac{\mu}{\delta}-C^{*}\right)-\gamma_{1}\left(C^{*}\right)-(\alpha+\delta)=f^{\prime}\left(C^{*}\right)<0 .
\end{aligned}
$$

Thus, the claimed result follows from the Lyapunov stability theorem [13].

\subsection{Global Stability}

Lemma 1. System (2) has no periodic orbit.

Proof. Let

$$
\begin{aligned}
h_{1}(I, C)= & \mu_{2}+\frac{\beta_{2} \mu}{\delta}+\left(\frac{\beta_{1} \mu}{\delta}-\beta_{2}-\gamma_{2}-\delta\right) I-\beta_{2} C \\
& -\beta_{1} I^{2}-\beta_{1} I C-\gamma_{1}(C) I, \\
h_{2}(I, C)= & \mu_{3}+\gamma_{1}(C)\left(\frac{\mu}{\delta}-C\right)-(\alpha+\delta) C, \\
D(I, C)= & \frac{1}{I C} .
\end{aligned}
$$

It can be obtained in the interior of $\Omega$ that

$$
\begin{aligned}
\frac{\partial\left(D h_{1}\right)}{\partial I}+\frac{\partial\left(D h_{2}\right)}{\partial C}= & -\frac{\beta_{1}}{C}-\frac{\gamma_{1}^{\prime}(C)}{I}-\frac{\mu_{2}}{I^{2} C}-\frac{\mu_{3}}{I^{2}}-\frac{\beta_{2}}{I^{2} C}\left(\frac{\mu}{\delta}-C\right) \\
& +\frac{\mu}{\delta I C^{2}}\left(\gamma_{1}^{\prime}(C) C-\gamma_{1}(C)\right) .
\end{aligned}
$$

Let

$$
k(x)=\gamma_{1}^{\prime}(x) x-\gamma_{1}(x) .
$$

As $k(0)=0$ and $k^{\prime}(x)=\gamma_{1}^{\prime \prime}(x) x<0$ for all $x>0$, $k(C)<0$. Thus, we have $\left(\partial\left(D h_{1}\right) / \partial I\right)+\left(\partial\left(D h_{2}\right) / \partial C\right)<0$.

Hence, in the interior of $\Omega$, system (2) has no periodic orbit according to the Bendixson-Dulac criterion [13].

On the boundary of $\Omega$, let $(\widetilde{I}, \widetilde{C})$ denote an arbitrary point. Thus, three possibilities may occur.

Case 1: $0<\widetilde{C}<(\mu / \delta), \widetilde{I}=0$. Then, $\left.(\mathrm{d} I / \mathrm{d} t)\right|_{(\widetilde{I}, \widetilde{C})}=\mu_{2}+$ $\beta_{2}((\mu / \delta)-\widetilde{C})>0$.
Case 2: $0<\widetilde{I}<(\mu / \delta), \quad \widetilde{C}=0$. Then, $\left.\quad(\mathrm{d} C / \mathrm{d} t)\right|_{(\widetilde{I}, \widetilde{C})}=$ $\mu_{3}>0$.

Case $3: \widetilde{I}+\widetilde{C}=(\mu / \delta), \widetilde{C} \neq 0, \widetilde{I} \neq 0$. Hence,

$$
\left.\frac{\mathrm{d}(I+C)}{\mathrm{d} t}\right|_{(\widetilde{I}, \widetilde{C})}=-\mu_{1}-\gamma_{2} \widetilde{I}-\alpha \widetilde{C}<0 .
$$

Thus, system (2) has no periodic orbit across the arbitrary point $(\widetilde{I}, \widetilde{C})$. The proof is completed.

In what follows, the main result of this paper will be given as follows.

Theorem 3. $E^{*}$ is globally asymptotically stable.

Proof. Based on Theorem 1, Lemma 1, and Theorem 2, the claimed result follows from the generalized Poincare-Bendixson theorem [13].

\section{Numerical Experiments}

To illustrate the main result of this paper and the impacts of different countermeasure probabilities on viral spread, some numerical experiments are presented in this section.

Example 1. Consider system (1) with $\mu_{1}=0.55, \mu_{2}=0.25$, $\mu_{3}=0.2, \alpha=0.02, \beta_{1}=0.05, \beta_{2}=0.03, \gamma_{2}=0.02, \delta=0.1$, and $\gamma_{1}(C)=0.05 C /(1+C)$. The initial condition is $(S(0), I(0), C(0))=(3,1,5)$. In Figure 2, a comparison between the new proposed SICS model and the original SICS model is shown, from which it can be seen that the new proposed model is more reasonable in predicting virus prevalence because computer viruses would not go extinct (i.e., $I \geq 1$ ), which demonstrates that the linear countermeasure probability overestimates the suppression of countermeasures on virus diffusion when compared to the nonlinear one.

Example 2. Consider system (1) with $\mu_{1}=55, \mu_{2}=38$, $\mu_{3}=7, \alpha=0.01, \beta_{1}=0.52, \beta_{2}=0.015, \gamma_{2}=0.01, \delta=0.02$, and $\gamma_{1}(C)=0.006 C^{0.15}$. Six different initial conditions are listed below.

(1) $(S(0), I(0), C(0))=(325,25,10)$.

(2) $(S(0), I(0), C(0))=(925,125,90)$.

(3) $(S(0), I(0), C(0))=(1525,225,170)$.

(4) $(S(0), I(0), C(0))=(2125,325,250)$. 


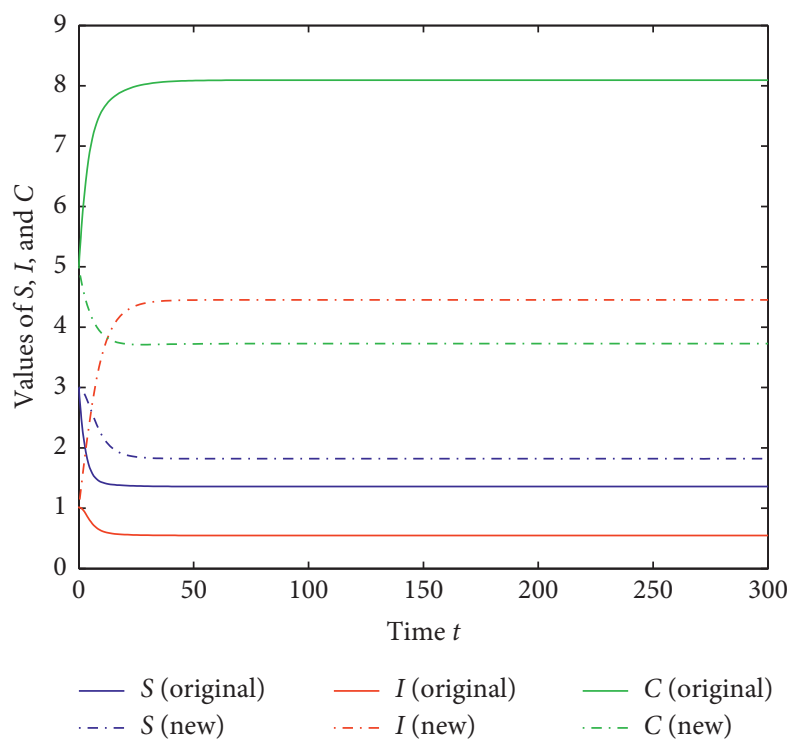

FIGURE 2: A comparison between the new proposed SICS model and the original SICS model.

(5) $(S(0), I(0), C(0))=(2725,425,330)$.

(6) $(S(0), I(0), C(0))=(3325,525,410)$.

Figure 3 shows six orbits of system (1) with different initial conditions for a common system. It can be seen from this figure that no matter where the initial state starts, computer viruses would always exist and tend to a steady state, which coincides with the main result. This also reveals that the global stability is independent of the initial state.

Example 3. Consider system (1) with the common initial condition $(S(0), I(0), C(0))=(1050,450,105)$, and six sets of parameters are given in Table 1 . Six orbits of system (1) with different system parameters for a common initial condition are shown in Figure 4, from which it can be seen that computer viruses would remain present and tend to a steady state, which accords with the main result. Additionally, this figure reveals that even starting from the same initial state the system would approach to different states for different parameters, which is distinct from the phenomenon in Example 2.

Example 4. Consider system (1) with $\mu_{1}=5.5, \mu_{2}=3.8$, $\mu_{3}=0.7, \alpha=0.01, \beta_{1}=0.52, \beta_{2}=0.015, \quad \gamma_{2}=0.01$, and $\delta=0.02$. The initial condition is $(S(0), I(0), C(0))=$ $(325,25,10)$. Figure 5 shows the influences of the varied countermeasure probabilities on the number of infected computers, where $\gamma_{1}(C)=0.08 C^{0.2}, \gamma_{2}(C)=(0.08 C /(1+$ $0.416 C))$, and $\gamma_{3}(C)=(0.08 C /(1+C))$. This figure also demonstrates that the nonlinear countermeasure probabilities which are continuously differentiable up to the second order may have many forms and pose different impacts on viral spread.

\section{Summary and Outlook}

A new SICS model has been proposed and analyzed in this paper. The global stability of the unique (viral) equilibrium

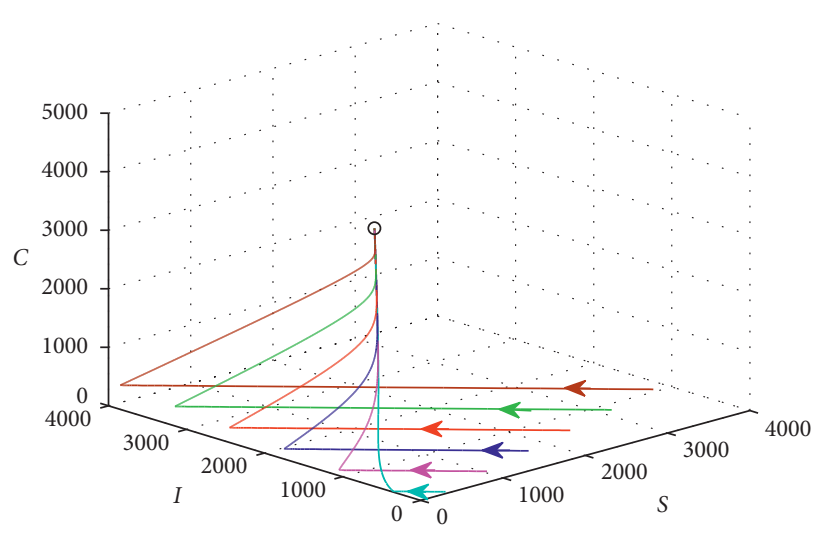

FIGURE 3: Six orbits of system (1) with different initial conditions.

has been proved and illustrated completely. Besides, a comparison between the new proposed model and the original SICS model has been shown, and the effects of varied countermeasure probabilities have also been revealed. The numerical experiments demonstrate that the nonlinear countermeasure probability is more reasonable than the linear one.

Additionally, the follow-up work arrangement is as follows. Firstly, time delays (e.g., [14, 15]), pulses (e.g., [16]), random fluctuations (e.g., $[17,18]$ ), and optimal control strategies (e.g., [19]) can be considered in the new model. Secondly, the new model may be extended on wireless sensor networks (e.g., [20-22]). With the popularity of social networks, individuals' participation has a particularly important effect on information diffusion including propagation of computer viruses. For example, Alduaiji et al. [23] developed an influence propagation model for clique-based community detection in social networks. Li et al. [24] proposed a metric to measure the community-diversified influence in social networks. Therefore, the new model may also be extended in social networks. Finally, the new 
TABLE 1: The parameters of system (1) for Example 3.

\begin{tabular}{lcccccc}
\hline Parameter & Set 1 & Set 2 & Set 3 & Set 4 & Set 5 & Set 6 \\
\hline$\mu_{1}$ & 55 & 35 & 25 & 15 & 10 & 18 \\
$\mu_{2}$ & 38 & 15 & 25 & 25 & 20 & 19 \\
$\mu_{3}$ & 7 & 10 & 5 & 0.03 & 0.02 & 0.02 \\
$\alpha$ & 0.01 & 0.03 & 0.22 & 0.72 & 0.62 \\
$\beta_{1}$ & 0.52 & 0.52 & 0.01 & 0.08 & 0.18 \\
$\beta_{2}$ & 0.015 & 0.13 & 0.03 & 0.02 & 0.04 \\
$\gamma_{2}$ & 0.01 & 0.02 & 0.02 & 0.03 & 0.02 \\
$\delta$ & 0.02 & $0.01 C^{0.3}$ & $0.1 C^{0.1}$ & $0.01 C^{0.5}$ & $0.03 C^{0.25}$ \\
$\gamma_{1}(C)$ & $0.06 C^{0.15}$ & & & 0.02 \\
\hline
\end{tabular}

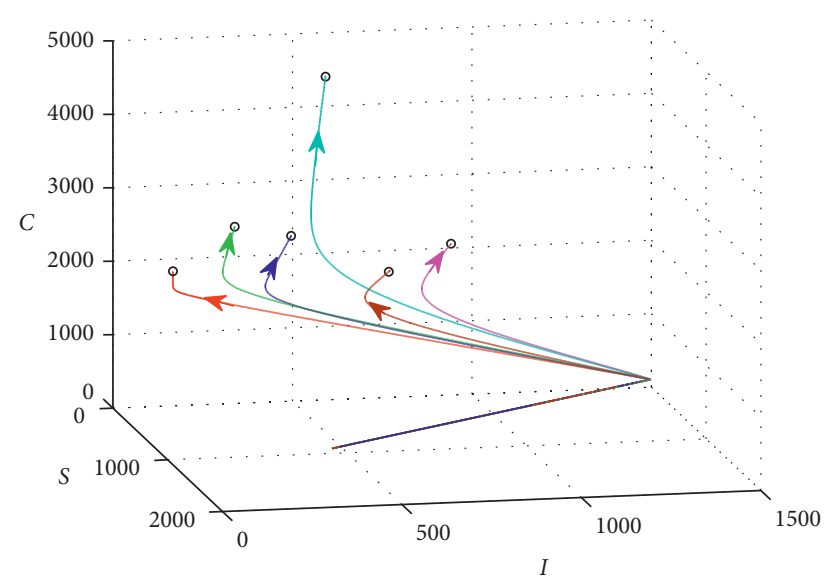

FIGURE 4: Six orbits of system (1) with different system parameters.

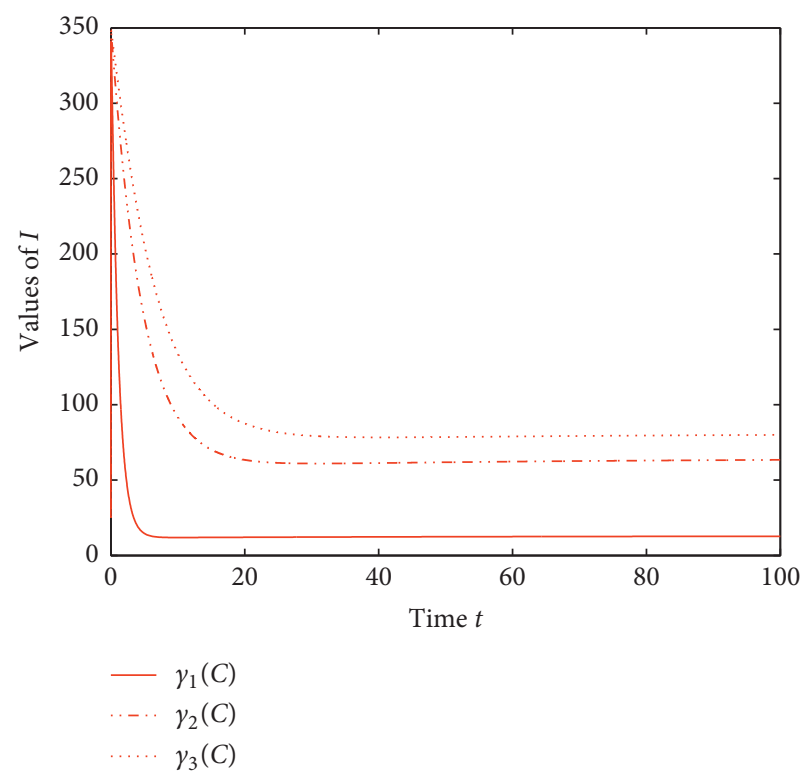

Figure 5: The impacts of the varied countermeasure probabilities on $I$.

proposed model can be formulated for cloud computing security (e.g., [25]).

\section{Data Availability}

The data included in this study are available from the corresponding author upon request.

\section{Conflicts of Interest}

The authors declare that there are no conflicts of interest with regard to the publication of this paper.

\section{Authors' Contributions}

Xulong Zhang conceived and designed the study and reviewed and edited the manuscript. Yong Li performed the numerical experiments. All authors read and approved the manuscript.

\section{Acknowledgments}

This study was supported by the Natural Science Foundation of Shanxi Province of China under grant nos. 201801 D121117 and 201901D111311.

\section{References}

[1] P. Szor, The Art of Computer Virus Research and Defense, Addison-Wesley Professional, Boston, MA, USA, 2005.

[2] J. C. Wierman and D. J. Marchette, "Modeling computer virus prevalence with a susceptible-infected-susceptible model with reintroduction," Computational Statistics \& Data Analysis, vol. 45, no. 1, pp. 3-23, 2004.

[3] A. d'Onofrio, "A note on the global behaviour of the networkbased SIS epidemic model," Nonlinear Analysis Real World Applications, vol. 9, no. 4, pp. 1567-1572, 2008.

[4] B. K. Mishra and N. Jha, "Fixed period of temporary immunity after run of anti-malicious software on computer nodes," Applied Mathematics and Computation, vol. 190, no. 2, pp. 1207-1212, 2007.

[5] C. Gan, Q. Feng, Q. Zhu, Z. Zhang, Y. Zhang, and Y. Xiang, "Analysis of computer virus propagation behaviors over complex networks: a case study of oregon routing network," Nonlinear Dynamics, vol. 100, no. 2, pp. 1725-1740, 2020.

[6] L.-X. Yang, X. Yang, L. Wen, and J. Liu, "A novel computer virus propagation model and its dynamics," International Journal of Computer Mathematics, vol. 89, no. 17, pp. 23072314, 2012.

[7] Q. Zhu, X. Yang, L. Yang, and X. Zhang, "A mixing propagation model of computer viruses and countermeasures," Nonlinear Dynamics, vol. 73, no. 7, pp. 1433-1441, 2013.

[8] L.-X. Yang and X. Yang, "The effect of infected external computers on the spread of viruses: a compartment modeling study," Physica A: Statistical Mechanics and Its Applications, vol. 392, no. 24, pp. 6523-6535, 2013. 
[9] X. Zhang and C. Gan, "Global attractivity and optimal dynamic countermeasure of a virus propagation model in complex networks," Physica A: Statistical Mechanics and Its Applications, vol. 490, pp. 1004-1018, 2018.

[10] W. Liu and S. Zhong, "Web malware spread modelling and optimal control strategies," Scientific Reports, vol. 7, no. 1, 2017.

[11] L.-C. Chen and K. M. Carley, "The impact of countermeasure propagation on the prevalence of computer viruses," IEEE Transactions on Systems, Man and Cybernetics, Part B (Cybernetics), vol. 34, no. 2, pp. 823-833, 2004.

[12] H. R. Thieme, "Convergence results and a poincare-bendixson trichotomy for asymptotically autonomous differential equations," Journal of Mathematical Biology, vol. 30, no. 7, pp. 755-763, 1992.

[13] R. C. Robinson, An Introduction to Dynamical System: Continuous and Discrete, Prentice Hall, Englewood Cliffs, NJ, USA, 2004.

[14] C. Zhang, W. Liu, J. Xiao, and Y. Zhao, "Hopf bifurcation of an improved SLBS model under the influence of latent period," Mathematical Problems in Engineering, vol. 2013, Article ID 196214, 2013.

[15] J. Ren and Y. Xu, "Stability and bifurcation of a computer virus propagation model with delay and incomplete antivirus ability," Mathematical Problems in Engineering, vol. 2014, Article ID 475934, 2014.

[16] Y. Yao, X. D. Feng, W. Yang, W. L. Xiang, and F. X. Gao, "Analysis of a delayed Internet worm propagation model with impulsive quarantine strategy," Mathematical Problems in Engineering, vol. 2014, Article ID 369360, 2014.

[17] C. Zhang, Y. Zhao, Y. Wu, and S. Deng, "A stochastic dynamic model of computer viruses," Discrete Dynamics in Nature and Society, vol. 2012, Article ID 264874, 2012.

[18] J. Amador, "The stochastic SIRA model for computer viruses," Applied Mathematics and Computation, vol. 232, pp. 11121124, 2014.

[19] C. Zhang and H. Huang, "Optimal control strategy for a novel computer virus propagation model on scale-free networks," Physica A: Statistical Mechanics and Its Applications, vol. 451, pp. 251-265, 2016.

[20] L. Feng, L. Song, Q. Zhao, and H. Wang, "Modeling and stability analysis of worm propagation in wireless sensor network," Mathematical Problems in Engineering, vol. 2015, Article ID 129598, 2015.

[21] A. Singh, A. K. Awasthi, K. Singh, and P. K. Srivastava, "Modeling and analysis of worm propagation in wireless sensor networks," Wireless Personal Communications, vol. 98, no. 3, pp. 2535-2551, 2018.

[22] Z. Zhang, S. Kundu, and R. Wei, "A delayed epidemic model for propagation of malicious codes in wireless sensor network," Mathematics, vol. 7, no. 5, Article ID 396, 2019.

[23] N. Alduaiji, A. Datta, and J. Li, "Influence propagation model for clique-based community detection in social networks," IEEE Transactions on Computational Social Systems, vol. 5, no. 2, pp. 563-575, 2018.

[24] J. Li, T. Cai, K. Deng, X. Wang, T. Sellis, and F. Xia, "Community-diversified influence maximization in social networks," Information Systems, vol. 92, Article ID 101522, 2020.

[25] C. Gan, Q. Feng, X. Zhang, Z. Zhang, and Q. Zhu, "Dynamical propagation model of malware for cloud computing security," IEEE Access, vol. 8, pp. 20325-20333, 2020. 